\title{
Rezidiv eines systemischen, diffus-großzelligen B-Zell-Lymphoms unter dem Bild eines primär kutanen, diffus-großzelligen B-Zell-Lymphoms, Leg Type
}

\author{
Relapse of a Systemic Diffuse Large B-Cell-Lymphoma Looking Like a Primary Cutaneous Diffuse Large \\ B-Cell-Lymphoma, Leg Type
}

Autoren

Institut

\section{T. Vogel, E. Schultz}

Hautklinik am Klinikum Nürnberg Nord

\section{Bibliografie}

Dol http://dx.doi.org/ 10.1055/s-0030-1256671 Akt Dermatol 2011; 37: 261-263 @ Georg Thieme Verlag KG Stuttgart · New York ISSN 0340-2541

Korrespondenzadresse Tobias Vogel

Hautklinik am Klinikum Nürnberg Nord Prof.-Ernst-Nathan-Str. 1 90419 Nürnberg Tobias.Vogel@ klinikum-nuernberg.de

\section{Zusammenfassung $\nabla$}

Wir berichten über eine 77-jährige Patientin, welche sich mit dem klinischen Bild eines Erysipels des rechten Unterschenkels vorstellte. Anamnestisch war bei ihr ein hochmalignes, CD20positives, diffus-großzelliges B-Zell-Lymphom mit pulmonalem und kutanem Befall bekannt, welches nach einer Polychemotherapie für acht Monate in kompletter Remission verblieb. Nach Rückbildung der durch das Erysipel verursachten Schwellung kamen ausgedehnte, zum Teil ulzerierte Knoten zum Vorschein, welche sich histologisch und immunhistochemisch als Infiltrate eines CD20-positiven, diffus-großzelligen B-ZellLymphoms erwiesen. Neben dem kutanen Befall waren auch die regionären Lymphknoten betroffen. Der vorgestellte Fall zeigt, dass ein vormals systemisches, diffus-großzelliges B-Zell-Lymphom sich in der Rezidivsituation als primär kutanes, diffus-großzelliges B-Zell-Lymphom, leg type, präsentieren kann. Zieht man die häufige systemische Ausbreitung dieses Lymphoms in Betracht, so stellt sich die Frage, ob es sich bei diesem Lymphom wirklich um ein primär kutanes Lymphom oder eher um ein systemisches Lymphom mit bevorzugtem Hautbefall handelt.

\section{Einleitung}

$\nabla$

Das diffus-großzellige B-Zell-Lymphom des Beines (primary cutaneous diffuse large B-cell lymphoma, leg type, PCDLBL, LT) zeichnet sich durch das Auftreten von rötlichen bis bläulichen Knoten und Tumoren im Bereich der unteren Extremität bei älteren Patienten aus [1-4]. Aufgrund des häufig vorkommenden extrakutanen Befalls ist die Prognose mit einer 5-Jahres-Überlebensrate von $55 \%$ erheblich schlechter als bei den indolenten kutanen B-Zell-Lymphomen [5]. In der aktuellen WHO-EORTC-Lymphom-Klassifikation der kutanen Lymphome werden neben dem PCDLBL, LT, auch noch andere Formen des primär kutanen, diffus-großzelligen B-Zell-Lymphoms aufgeführt (PCDLBL, other) [1,2,6]. Dabei handelt es sich um diffuse B-Zell-Lymphome, welche sich klinisch, histologisch und phänotypisch nicht eindeutig dem PCDLBL, LT, bzw. dem primär kutanen Keimzentrumslymphom zuordnen lassen $[1,2,6]$.

\section{Anamnese \\ $\nabla$}

Eine 77-jährige Patientin wurde aufgrund einer seit ca. 4 Wochen zunehmenden, stark schmerzhaften Schwellung, Rötung und Überwärmung des rechten Unterschenkels stationär aufgenommen.

\section{Dermatologischer Befund}

Der rechte Unterschenkel zeigte sich gerötet, geschwollen und überwärmt. Zentral imponierte ein $10 \times 20 \mathrm{~cm}$ großes erosives Areal, teils schmierig belegt.

\section{Labor}

CRP $25 \mathrm{mg} / \mathrm{dl}($ Norm $<0,5)$

$\mathrm{Hb} 9,5 \mathrm{~g} / \mathrm{dl}$ (Norm 12,0-16,0)

Hämatokrit 29,1 g/dl (Norm 36,0-46,0)

Erythrozyten 3,35/pl (Norm 4,20-5,40)

Leukozyten, MCV, MCH, MCHC unauffällig

\section{CT-Thorax}

Kein intrathorakaler Tumor-Nachweis bei postentzündlichen Veränderungen in beiden Unterlappen. 


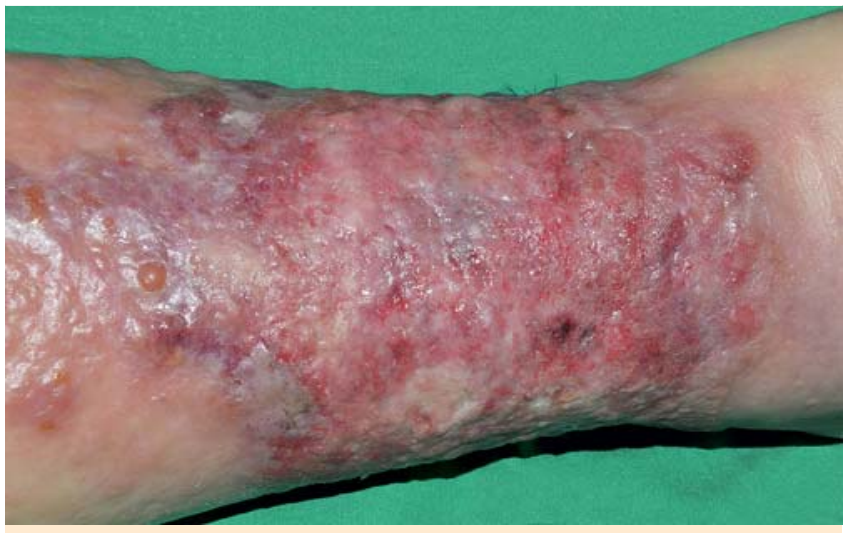

Abb. 1 Klinischer Befund nach Rückgang der Schwellung des Erysipels.

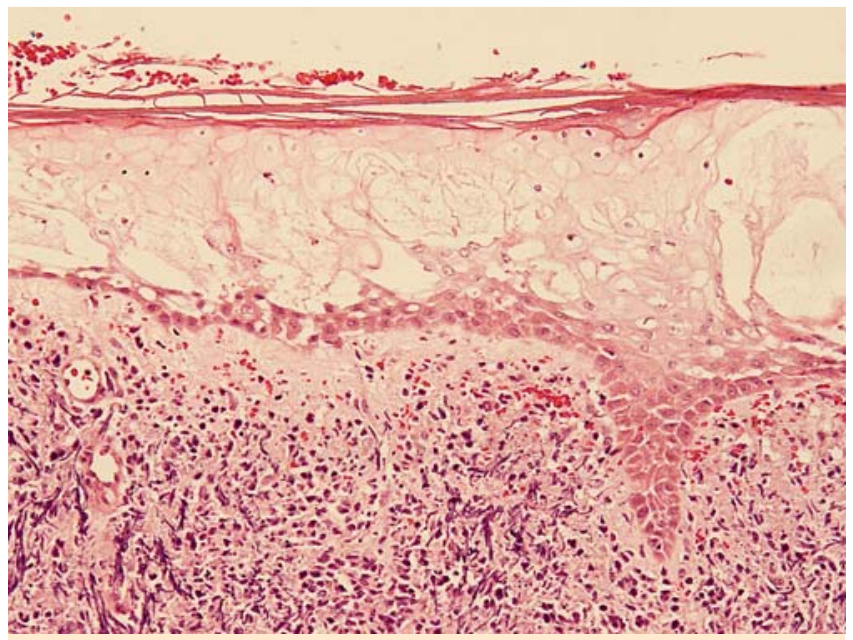

Abb. 2 HE-Färbung: Dermales Infiltrat mäßig pleomorpher Lymphomzellen mit zahlreichen Mitosefiguren.

\section{CT-Abdomen}

Ausgedehnte Lymphadenopathie inguinal rechts (bis $48 \times 38 \mathrm{~mm})$, an der Iliaca-externa-Gruppe rechts $(25 \times 35 \mathrm{~mm})$, an der Iliaca-communis-Gruppe sowie paraaortal rechts in Höhe der Nierenetage $(16 \times 14 \mathrm{~mm})$. Kein fokaler Herdbefund an Leber, Milz, Nieren, Pankreas und Nebennieren.

\section{Histologie \\ $\nabla$}

Unterhalb einer von der Basis intraepidermal pseudovesikulär abgehobenen Epidermis findet sich ein ausgedehntes dermales Infiltrat durch mäßig pleomorphe Lymphomzellen, die zahlreiche Mitosefiguren zeigen. Diese Infiltrate setzen sich kontinuierlich bis in die Tiefe fort. Immunhistologisch ergeben sich innerhalb der Tumorzellaggregate kräftige Positivitäten für die B-Zellmarker CD79 und CD20, hingegen CD8 und CD4 negativ. Der Proliferationsmarker MIB-1 in über $80 \%$ der Tumorzellkerne positiv exprimiert, CD30 mit einer ebenfalls kräftigen Koexpression in herdförmig bis zu 30\% der Tumorzellkerne. Bcl-2 durchgängig positiv, CD10 negativ, EBV (EBER) negativ $(\bullet$ Abb. $2-4)$.

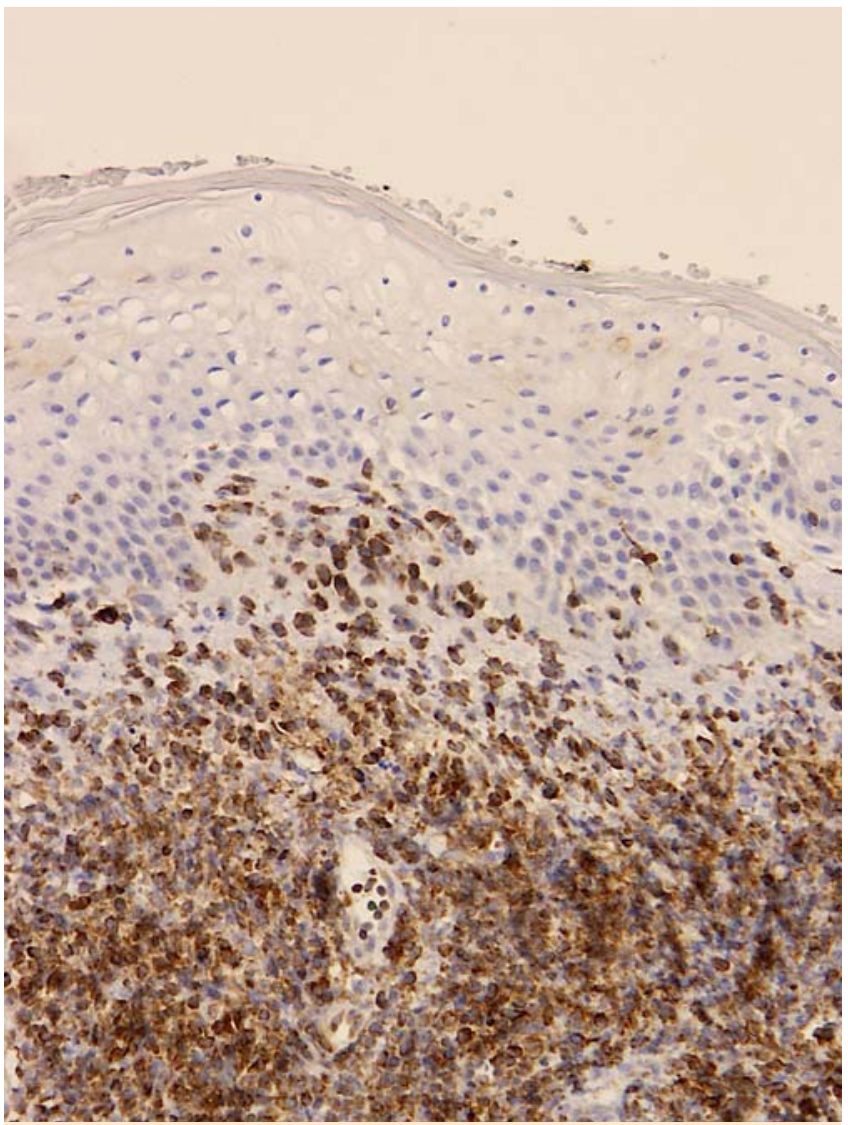

Abb. 3 Positivität für $\mathrm{BCL}-2$

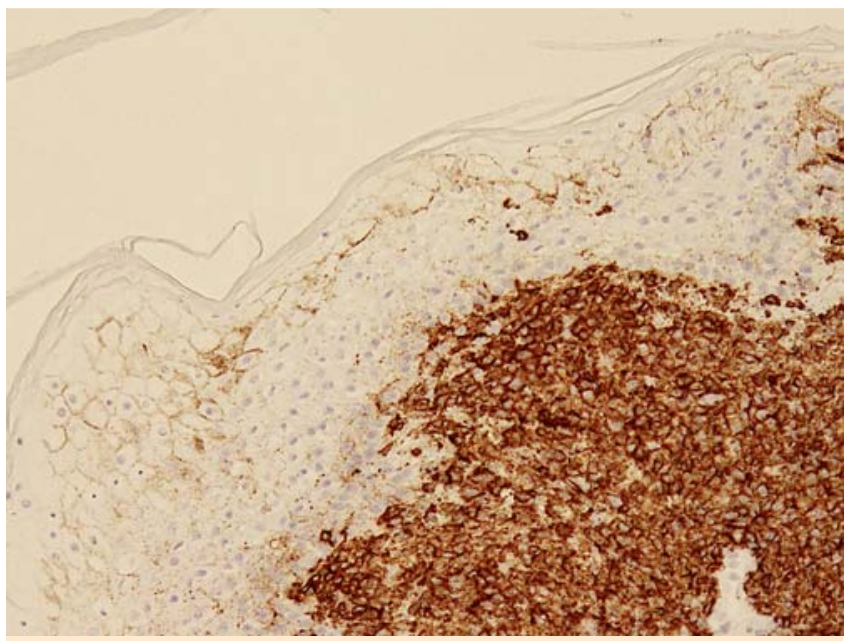

Abb. 4 Positivität für CD20.

\section{Therapie und Verlauf}

$\nabla$

Unter einer i.v. Antibiose mit initial Cefuroxim $(3 \times 1,5 \mathrm{~g}$ tgl.) und im weiteren Verlauf Umstellung auf Piperacillin $4 \mathrm{~g}$ und Tazobactam $1 \mathrm{~g} 3 \times$ tgl. kam es zu einer kontinuierlichen Befundbesserung mit Regredienz der Rötung und Schwellung.

Nachdem die initiale Schwellung rückläufig war, imponierten jetzt derbe, knotige Veränderungen ( $\bullet$ Abb. 1).

Histologisch zeigten sich Infiltrate eines diffus-großzelligen BZell-Lymphoms ( $\odot$ Abb. 2-4). In der Durchuntersuchung fand sich eine Beteiligung der regionären Lymphknoten. Unter einer Chemotherapie mit sechs Zyklen nach dem R-CHOP-14-Schema 
und anschließend zwei Zyklen Rituximab-Monotherapie konnte eine komplette Remission erreicht werden, am Bein zeigten sich lediglich narbige Residuen [2,7]. Sieben Monate nach Beendigung der Therapie erlitt die Patientin ein Rezidiv im Musculus triceps brachii links mit peripherer Neuropathie des N. radialis. Nach Radiatio kam es hier zu einer Remission, jedoch wurde aktuell eine neue Raumforderung im linken Oberschenkel gefunden, die noch weiter abgeklärt wird.

\section{Diskussion}

11/2008 trat erstmals bei der Patientin ein diffus-großzelliges BZell-Lymphom mit kutanem Befall in der Schulterregion und diffus-pulmonalem Befall auf, welches vermutlich aus einem bereits vorher bekannten Immunozytom durch Transformation entstanden war [8]. Nach Vortherapie mit Vincristin und Prednison sowie anschließend 4 Zyklen Chemotherapie mit Bendamustin, Mitoxantron und Rituximab kam es 04/2009 zu einer kompletten Remission [3,7].

12/2009 kam es dann zu einem Rezidiv unter dem klinischen Bild eines primär kutanen B-Zell-Lymphoms des Beines (PCDLBL, LT). Im hier vorliegenden Fall ist aufgrund des histologischen Bildes und des immunhistochemischen Markerstatus von einem Rezidiv des ein Jahr zuvor aufgetretenen diffus-großzelligen BZell-Lymphoms auszugehen. Es ist bekannt, dass ein PCDLBL, LT, in ein systemisches Lymphom übergehen kann $[4,5,7]$. Bei unserer Patientin war es umgekehrt, ein primär systemisches B-ZellLymphom rezidivierte unter dem klinischen Bild eines PCDLBL, LT. Unser Fallbericht verdeutlicht, dass es sich bei dieser Lymphomentität wohl in der Mehrzahl der Fälle um eine systemische Erkrankung mit einer bevorzugten Hautbeteiligung handeln dürfte. Dies sollte auch beim diagnostischen und therapeutischen Vorgehen berücksichtigt werden.

\section{Abstract}

\section{Relapse of a Systemic Diffuse Large B-Cell-Lymphoma Looking Like a Primary Cutaneous Diffuse Large B-Cell-Lymphoma, Leg Type}

We report on a 77-year old female patient who introduced herself with an erysipelas on her right calf. In the past she had suffered from a diffuse large B-cell-lymphoma with pulmonary and cutaneous manifestations. A complete remission had been achieved by polychemotherapy 8 month ago.

After regression of the oedema caused by the erysipelas, numerous, partly ulcerated nodes were visible. Histology and immunohistochemistry revealed cutaneous infiltrates of a CD-20-positive diffuse large B-cell-lymphoma. Besides the cutaneous lesion, also her regional lymph nodes were affected.

This case shows that a previously systemic diffuse large B-celllymphoma can mimick a primary cutaneous diffuse large B-celllymphoma, leg type. With regard to the frequent systemic involvement of the latter type of lymphoma, the question arises whether this is really a primary cutaneous lymphoma but rather a systemic lymphoma with a predilection for cutaneous infiltrates.

\section{Literatur}

1 Grange $F$ et al. Primary cutaneous diffuse large B-cell-lymphoma, leg type. Arch Dermatol 2007; 143: 1144-1150

2 Stadler $R$. Kutane Lymphome - Klassifikationen und stadiengerechte Therapieoptionen. Hautarzt 2006; 57: $744-755$

3 Gellrich S, Muche JM, Audring H, Sterry W. Kutane B-Zell-Lymphome. Hautarzt 2000; 51: $363-375$

4 Plettenberg $H$, Hilton S, Stege $H$ et al. Großzelliges B-Zell-Lymphom des Unterschenkels. Hautarzt 2004; 55: 395 - 397

5 Chen YF, Li YC, Chen LM et al. Primary cutaneous diffuse large B cell lymphoma relapsed solely as a huge lung tumor mimicking a primary pulmonary lymphoma. Int J Hematol 2009; 91 (1): 112 - 116

6 Storz MN, van de Rijn M, Kim YH et al. Gene expression profiles of cutaneous B cell lymphoma. J Invest Dermatol 2003; 120: 865-870

7 Heinzerling LM, Urbanek M, Funk JO et al. Reduction of tumor burden and stabilization of disease by systemic therapy with anti-CD20 antibody (rituximab) in patients with primary cutaneous B-cell lymphoma. Cancer 2000; 89 (8): 1835-1844

8 Feichter G, Dalquen P. Zytopathologie. 2. Aufl. Berlin: Springer; 1999: 359 